TRANSACTIONS OF THE

AMERICAN MATHEMATICAL SOCIETY

Volume 363, Number 7, July 2011, Pages 3621-3637

S 0002-9947(2011)05228-1

Article electronically published on February 7, 2011

\title{
LINEAR $\sigma$-ADDITIVITY AND SOME APPLICATIONS
}

\author{
TAL ORENSHTEIN AND BOAZ TSABAN
}

\begin{abstract}
We show that countable increasing unions preserve a large family of well-studied covering properties, which are not necessarily $\sigma$-additive. Using this, together with infinite-combinatorial methods and simple forcing theoretic methods, we explain several phenomena, settle problems of Just, Miller, Scheepers and Szeptycki (1996), Gruenhage and Szeptycki (2005), Tsaban and Zdomskyy (2008), and Tsaban (2006), (2007), and construct topological groups with very strong combinatorial properties.
\end{abstract}

\section{INTRODUCTION}

The following natural definition unifies all results presented here.

Definition 1.1. Let $\mathcal{F}$ be a family of topological spaces. $\mathcal{F}$ is linearly $\sigma$-additive if it is preserved by countable increasing unions. That is: For each topological space $X=\bigcup_{n} X_{n}$ with $X_{1} \subseteq X_{2} \subseteq \ldots$ and $X_{n} \in \mathcal{F}$ for all $n \in \mathbb{N}, X \in \mathcal{F}$.

Removing the restriction that $X_{n} \subseteq X_{n+1}$ for all $n$, we obtain the definition of a $\sigma$-additive family. We identify a topological property with the family of all topological spaces satisfying it. Thus, we may talk about linearly $\sigma$-additive properties.

We consider additivity in the context of topological selection principles, to which we now give a brief introduction 1 This is a framework suggested by Scheepers in 24] to study in a uniform manner a variety of properties introduced in different mathematical disciplines, since the early 1920s, by Menger, Hurewicz, Rothberger, and Gerlits and Nagy, and many others.

Let $X$ be a topological space. We say that $\mathcal{U}$ is a cover of $X$ if $X=\bigcup \mathcal{U}$ but $X \notin \mathcal{U}$. Often, $X$ is considered as a subspace of another space $Y$, and in this case we always consider covers of $X$ by subsets of $Y$, and require instead that no member of the cover contains $X$. Let $\mathrm{O}(X)$ be the family of all open covers of $X$. Define the following subfamilies of $\mathrm{O}(X): \mathcal{U} \in \Omega(X)$ if each finite subset of $X$ is contained in some member of $\mathcal{U} . \mathcal{U} \in \Gamma(X)$ if $\mathcal{U}$ is infinite, and each element of $X$ is contained in all but finitely many members of $\mathcal{U}$.

Some of the following statements may hold for families $\mathscr{A}$ and $\mathscr{B}$ of covers of $X$ :

$\mathrm{S}_{1}(\mathscr{A}, \mathscr{B}):$ For all $\mathcal{U}_{1}, \mathcal{U}_{2}, \ldots \in \mathscr{A}$, there are $U_{1} \in \mathcal{U}_{1}, U_{2} \in \mathcal{U}_{2}, \ldots$ such that $\left\{U_{n}: n \in \mathbb{N}\right\} \in \mathscr{B}$.

$\mathrm{S}_{\text {fin }}(\mathscr{A}, \mathscr{B}):$ For all $\mathcal{U}_{1}, \mathcal{U}_{2}, \ldots \in \mathscr{A}$, there are finite $\mathcal{F}_{1} \subseteq \mathcal{U}_{1}, \mathcal{F}_{2} \subseteq \mathcal{U}_{2}, \ldots$ such that $\bigcup_{n} \mathcal{F}_{n} \in \mathscr{B}$.

Received by the editors August 2, 2009 and, in revised form, October 20, 2009.

2010 Mathematics Subject Classification. Primary 54D20, 54D80, 54H11; Secondary 03E17, $03 \mathrm{E} 35$.

${ }^{1}$ Extended introductions to this field are available in [19, 27, 31]. 
$\mathrm{U}_{\text {fin }}(\mathscr{A}, \mathscr{B}):$ For all $\mathcal{U}_{1}, \mathcal{U}_{2}, \ldots \in \mathscr{A}$, none containing a finite subcover, there are finite $\mathcal{F}_{1} \subseteq \mathcal{U}_{1}, \mathcal{F}_{2} \subseteq \mathcal{U}_{2}, \ldots$ such that $\left\{\bigcup \mathcal{F}_{n}: n \in \mathbb{N}\right\} \in \mathscr{B}$.

We say, e.g., that $X$ satisfies $\mathrm{S}_{1}(\mathrm{O}, \mathrm{O})$ if the statement $\mathrm{S}_{1}(\mathrm{O}(X), \mathrm{O}(X))$ holds. This way, $\mathrm{S}_{1}(\mathrm{O}, \mathrm{O})$ is a property of topological spaces, and similarly for all other statements and families of covers. Under some mild hypotheses on the considered topological spaces, each nontrivial property among these properties, where $\mathscr{A}, \mathscr{B}$ range over $\mathrm{O}, \Omega, \Gamma$, is equivalent to one in Figure 1 [24, 17. In this diagram, an arrow denotes implication.

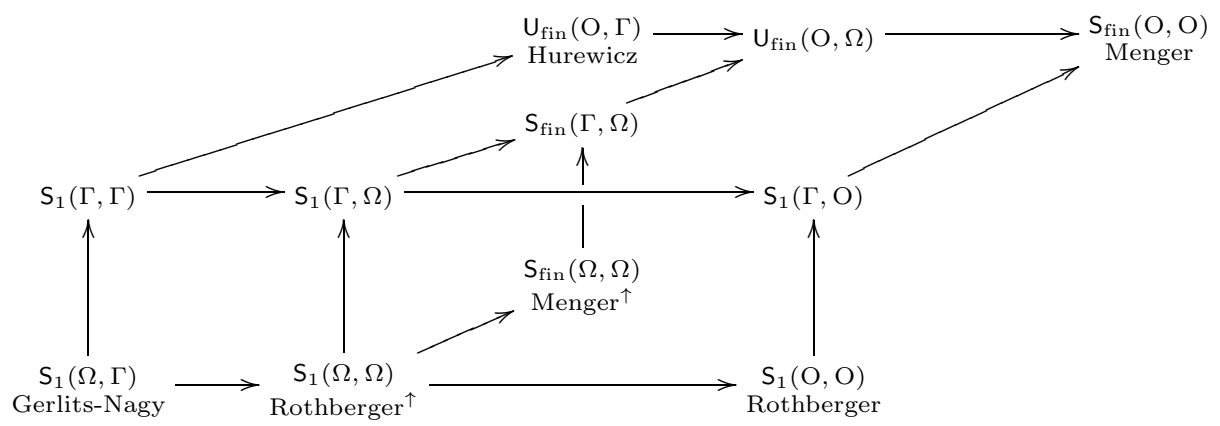

Figure 1. The Scheepers Diagram

In this diagram, the classical name of a property is indicated below it, as well as two names ending with a symbol $\uparrow$, by which we indicate that the properties $\mathrm{S}_{1}(\Omega, \Omega)$ and $\mathrm{S}_{\text {fin }}(\Omega, \Omega)$ may also be viewed as classical ones [23, 17, in accordance with the following notation.

Definition 1.2. Let $P$ be a property of topological spaces. $X$ satisfies $P^{\uparrow}$ if all finite powers $X^{k}$ of $X$ satisfy $P$.

The Scheepers Diagram is at the heart of the field of topological selection principles, and many additional - classical and new - properties are studied in relation to it. The reader is encouraged to consider his favorite properties in light of the results presented here.

In Section 2, we prove that all properties in the Scheepers Diagram are linearly $\sigma$-additive and are thus hereditary for $F_{\sigma}$ subsets. This solves a problem of Tsaban and Zdomskyy from [36].

A crucial part of the proof that the studied properties are linearly $\sigma$-additive is a recent theorem of F. Jordan [15. Miller asked in 22 whether $\mathrm{S}_{1}(\Omega, \Gamma)$ is linearly $\sigma$-additive. A negative solution would have solved the notorious GerlitsNagy problem [13]. Using a brilliant argument, Jordan proved that this is not the case. We give a direct version of Jordan's solution and provide some applications.

In Section 3. we use Jordan's method in a proof that if there is an unbounded family of cardinality $\aleph_{1}$ in the Baire space, then there is an uncountable set of real numbers satisfying $S_{1}(\Omega, \Gamma)$. This settles in the positive a problem from the seminal paper of Just, Miller, Scheepers, and Szeptycki [17. Indeed, our result is more general and also solves a problem of Gruenhage and Szeptycki 14.

In Section 4 we apply linear $\sigma$-additivity to study heredity of properties, answer a question of Zdomskyy, and suggest a simple revision of a question of Bukovský, 
Recław, and Repický, which makes it possible to answer it in the positive. (The problem, as originally stated, was answered in the negative by Miller [20].) This section also explains the phenomenon observed in [3, that none of the considered properties is hereditary in the open case, whereas in the Borel case some are and some are not hereditary.

In Section [5 we apply our results to construct topological groups with strong combinatorial properties, and solve problems from [32] and [35].

1.1. Generalizations. The results presented here require little or no assumptions on the topology of the studied spaces. However, they are interesting even when restricting attention to, e.g., metric spaces or even subsets of $\mathbb{R}$.

For concreteness, we present the results only for the types of covers mentioned above, but the proofs show that they hold for many additional types. In particular, define $\mathrm{B}, \mathrm{B}_{\Omega}, \mathrm{B}_{\Gamma}$ as $\mathrm{O}, \Omega, \Gamma$ were defined, replacing open cover by countable Borel cover. The properties thus obtained have rich history of their own 28]. Mild assumptions on $X$ imply that the considered open covers may be assumed to be countable, and this makes the Borel variants of the studied properties (strictly) stronger [28]. All of the results presented here also hold in the Borel case (after replacing open or closed by Borel). Moreover, unlike some of the results in the open case, none of the Borel variant requires any assumption on the topology of $X$.

\section{Linear $\sigma$-AdDitivity in the Scheepers Diagram}

One motivation for studying linear $\sigma$-additivity in the context of the Scheepers Diagram is an experimentally observed dichotomy concerning additivity of properties in this diagram: Each property is either provably $\sigma$-additive or not even provably finitely additive 2 In this section, we prove the following.

Theorem 2.1. All properties in the Scheepers Diagram (Figure 1) are linearly $\sigma$-additive.

It remains to notice the following.

Lemma 2.2. Each linearly $\sigma$-additive property is either $\sigma$-additive or not finitely additive.

Proof. $\bigcup_{n} X_{n}=\bigcup_{n}\left(\bigcup_{m \leq n} X_{m}\right)$.

Corollary 2.3. Each property in the Scheepers Diagram is either $\sigma$-additive or not additive.

Before proving Theorem 2.1, we point out two additional consequences. In Problem 4.9 of [36] and Problem 6.2 of [35], Tsaban and Zdomskyy ask whether $S_{1}(\Gamma, \Omega)$ and $\mathrm{S}_{\text {fin }}(\Gamma, \Omega)$ are hereditary for $F_{\sigma}$ subsets. We obtain a positive answer.

Corollary 2.4. All properties in the Scheepers Diagram are hereditary for $F_{\sigma}$ subsets.

Proof. These properties are easily seen to be hereditary for closed subsets [17, and countable unions of closed subsets can be presented as countable increasing unions of closed subsets. Apply Theorem 2.1.

\footnotetext{
${ }^{2}$ A survey of the involved results, with complete proofs, is available in 33 .
} 
The following was observed in the past for at least some properties in the Scheepers Diagram, each time using an ad-hoc argument.

Corollary 2.5. Let $P$ be a property in the Scheepers Diagram, and $X, D$ be subspaces of some topological space. If $X$ satisfies $P$ and $D$ is countable, then $X \cup D$ satisfies $P$.

Proof. In light of Theorem 2.1, it remains to observe that for each singleton $\{a\}$, $X \cup\{a\}$ satisfies $P$. This is not hard to verify.

Theorem 2.1 is proved in parts. The properties $\mathrm{S}_{1}(\mathrm{O}, \mathrm{O}), \mathrm{S}_{1}(\Gamma, \mathrm{O}), \mathrm{S}_{\mathrm{fin}}(\mathrm{O}, \mathrm{O})$, $\mathrm{S}_{1}(\Gamma, \Gamma)$, and $\mathrm{U}_{\text {fin }}(\mathrm{O}, \Gamma)$ are in fact $\sigma$-additive [17, 26] (see [33]).

Linear $\sigma$-additivity of $\mathrm{U}_{\text {fin }}(\mathrm{O}, \Omega)$ was proved in [36] for sets of reals. It also follows from the following.

Theorem 2.6. For all $\Pi \in\left\{\mathrm{S}_{1}, \mathrm{~S}_{\mathrm{fin}}, \mathrm{U}_{\text {fin }}\right\}$ and $\mathscr{A} \in\{\Gamma, \Omega, \mathrm{O}\}, \Pi(\mathscr{A}, \Omega)$ is linearly $\sigma$-additive.

Proof. We prove the theorem for $\Pi=\mathrm{S}_{\text {fin }}$, the other proofs being similar.

Assume that $X=\bigcup_{n} X_{n}$ is an increasing union, with each $X_{n}$ satisfying $\mathrm{S}_{\text {fin }}(\mathscr{A}, \Omega)$. Let $\mathcal{U}_{1}, \mathcal{U}_{2}, \ldots \in \mathscr{A}(X)$. We first exclude the trivial case: Assume that for infinitely many $n$, there are $m_{n}$ and elements $U_{m_{n}} \in \mathcal{U}_{m_{n}}$ such that $X_{n} \subseteq U_{m_{n}}$. As $X$ is not contained in any member of any $\mathcal{U}_{n}$ and the sets $X_{n}$ increase to $X$, we may if necessary thin out the sequence $m_{n}$ to make it increasing. Then $\left\{U_{m_{n}}: n \in \mathbb{N}\right\} \in \Omega(X)$, and this suffices.

Thus, we may assume that for all $n, k$, we have that $\mathcal{U}_{k} \in \mathscr{A}\left(X_{n}\right)$. Take a partition $\mathbb{N}=\bigcup_{n} I_{n}$ of $\mathbb{N}$ into infinite sets $I_{n}$. Fix $n$. As $X_{n}$ satisfies $\mathrm{S}_{\text {fin }}(\mathscr{A}, \Omega)$, there are finite $\mathcal{F}_{k} \subseteq \mathcal{U}_{k}, k \in I_{n}$, such that $\bigcup_{k \in I_{n}} \mathcal{F}_{k} \in \Omega\left(X_{n}\right)$. Then $\bigcup_{k \in \mathbb{N}} \mathcal{F}_{k} \in$ $\Omega(X)$.

The remaining property, $\mathrm{S}_{1}(\Omega, \Gamma)$, was treated by F. Jordan.

2.1. Jordan's Theorem and some applications. The following technical lemma will be useful in the proof of Jordan's Theorem below.

Lemma 2.7. Let $Y \subseteq X$ be such that $Y$ satisfies $\mathrm{S}_{1}(\Gamma, \Gamma)$. Assume that for each $n$,

(1) $\mathcal{U}_{n}$ is an infinite family of open subsets of $X$; and

(2) for each $y \in Y, y \in U$ for all but finitely many $U \in \mathcal{U}_{n}$.

Then there are infinite $\mathcal{V}_{1} \subseteq \mathcal{U}_{1}, \mathcal{V}_{2} \subseteq \mathcal{U}_{2}, \ldots$, such that for each $y \in Y, y \in \bigcap \mathcal{V}_{n}$ for all but finitely many $n$.

Proof. It may be the case that no subset of $\mathcal{U}_{n}$ is in $\Gamma(Y)$.

Case 1: For all but finitely many $n, \mathcal{V}_{n}=\left\{U \in \mathcal{U}_{n}: Y \subseteq U\right\}$ is infinite. Then the sets $\mathcal{V}_{n}$ thus defined are as required.

Case 2: Let $I$ be the set of all $n$ such that $\mathcal{V}_{n}=\left\{U \in \mathcal{U}_{n}: Y \subseteq U\right\}$ is infinite, and $J=\mathbb{N} \backslash I$. For each $n \in J, \mathcal{W}_{n}=\left\{U \in \mathcal{U}_{n}: Y \nsubseteq U\right\}$ is infinite, and thus $\mathcal{W}_{n} \in \Gamma(Y)$. As $J$ is infinite, there are by Theorem 15 of [34 infinite $\mathcal{V}_{n} \subseteq \mathcal{W}_{n}$, $n \in J$, such that each $y \in Y$ belongs to $\bigcap \mathcal{V}_{n}$ for all but finitely many $n$. (Briefly: By thinning out if needed, we may assume that each $\mathcal{W}_{n}$ is countable and that $\mathcal{W}_{n} \cap \mathcal{W}_{m}=\emptyset$ for $m \neq n$ 24. Apply $\mathrm{S}_{1}(\Gamma, \Gamma)$ to the countable family of all cofinite subsets of all $\mathcal{W}_{n}$ to obtain $\mathcal{V} \in \Gamma(Y)$. Let $\mathcal{V}_{n}=\mathcal{V} \cap \mathcal{W}_{n}$.) The sets $\mathcal{V}_{1}, \mathcal{V}_{2}, \ldots$ are as required. 
Theorem 2.8 (Jordan [15]). $\mathrm{S}_{1}(\Omega, \Gamma)$ is linearly $\sigma$-additive.

Proof. We give a direct proof, following what seems to be the essence of Jordan's arguments. The following statement can be deduced from Theorem 7 in [15].

Lemma 2.9. Let $X=\bigcup_{n} X_{n}$ be an increasing union, where each $X_{n}$ satisfies $\mathrm{S}_{1}(\Gamma, \Gamma)$. For all $\mathcal{U}_{1} \in \Gamma\left(X_{1}\right), \mathcal{U}_{2} \in \Gamma\left(X_{2}\right), \ldots$, there are infinite $\mathcal{V}_{1} \subseteq \mathcal{U}_{1}, \mathcal{V}_{2} \subseteq$ $\mathcal{U}_{2}, \ldots$, such that for each $x \in X, x \in \bigcap \mathcal{V}_{n}$ for all but finitely many $n$.

Proof. Step 1: By Lemma 2.7, we may thin out the families $\mathcal{U}_{n}$ so that they remain infinite, and each member of $X_{1}$ belongs to all but finitely many $\bigcap \mathcal{U}_{n}$. Let $\mathcal{V}_{1}=\mathcal{U}_{1}$.

Step 2: By the same lemma, we may further thin out the families $\mathcal{U}_{n}, n \geq 2$, so that they remain infinite, and each member of $X_{2}$ belongs to all but finitely many $\bigcap \mathcal{U}_{n}, n>1$. Let $\mathcal{V}_{2}=\mathcal{U}_{2}$.

Step $k$ : By Lemma 2.7, we may further thin out the families $\mathcal{U}_{n}, n \geq k$, so that they remain infinite, and each member of $X_{k}$ belongs to all but finitely many $\cap \mathcal{U}_{n}$, $n \geq k$. Let $\mathcal{V}_{k}=\mathcal{U}_{k}$.

The sets $\mathcal{V}_{1}, \mathcal{V}_{2}, \ldots$ are as required.

Now, let $X=\bigcup_{n} X_{n}$ be an increasing union, where each $X_{n}$ satisfies $\mathrm{S}_{1}(\Omega, \Gamma)$. Let $\mathcal{U}_{1}, \mathcal{U}_{2}, \ldots \in \Omega(X)$. As in the argument of the proof of Lemma 2.7, we may assume that $\mathcal{U}_{n} \in \Omega\left(X_{n}\right)$ for all $n$. As $X_{n}$ satisfies $\mathrm{S}_{1}(\Omega, \Gamma)$, we may thin out $\mathcal{U}_{n}$ so that $\mathcal{U}_{n} \in \Gamma\left(X_{n}\right) 3$ Apply Lemma 2.9] and pick for each $n$ some $U_{n} \in$ $\mathcal{V}_{n} \backslash\left\{U_{1}, \ldots, U_{n-1}\right\} .\left\{U_{n}: n \in \mathbb{N}\right\} \in \Gamma(X)$.

Let $C(X)$ be the family of continuous functions $f: X \rightarrow \mathbb{R} . C(X)$ has the Arhangel'skiu property $\alpha_{2}$ if the following holds for all $f_{m}^{n} \in C(X), n, m \in \mathbb{N}$ : If for each $n, \lim _{m \rightarrow \infty} f_{m}^{n}(x)=0$ for all $x \in X$, then there are $m_{n}$ such that $\lim _{n} f_{m_{n}}^{n}(x)=0$ for each $x \in X$.

For the same reason briefly mentioned in the proof of Lemma 2.7, one may require in the definition of $\alpha_{2}$ that there are infinite $I_{1}, I_{2}, \ldots \subseteq \mathbb{N}$ such that some (equivalently, any) enumeration of the countable set $\bigcup_{n}\left\{f_{m}^{n}(x): m \in I_{n}\right\}$ converges to 0 , for each $x \in X$. Indeed, this was Arhangel'skil's original definition of $\alpha_{2}$.

Definition 2.10. Let $X=\bigcup_{n} X_{n}$ be an increasing union. $C(X)$ is $\left\{X_{n}\right\}_{n \in \mathbb{N}}-\alpha_{2}$ if the following holds whenever $f_{m}^{n} \in C\left(X_{n}\right)$ for all $m, n$ : If for each $n, \lim _{m} f_{m}^{n}(x)=0$ for all $x \in X_{n}$, then there are infinite $I_{1}, I_{2}, \ldots \subseteq \mathbb{N}$, such that for each $x \in X$, $\lim _{n} \sup \left\{\left|f_{m}^{n}(x)\right|: m \in I_{n}\right\}=04$

Clearly, $\left\{X_{n}\right\}_{n \in \mathbb{N}^{-}} \alpha_{2}$ implies $\alpha_{2}$. The proof of Lemma 2.9. with minor modifications, yields the following new result about function spaces.

Theorem 2.11. Let $X=\bigcup_{n} X_{n}$ be an increasing union such that $C\left(X_{n}\right)$ is $\alpha_{2}$ for all $n$. Then $C(X)$ is $\left\{X_{n}\right\}_{n \in \mathbb{N}}-\alpha_{2}$.

Proof. Assume that $f_{m}^{n} \in C\left(X_{n}\right)$ for all $m, n$, and for each $n$, $\lim _{m} f_{m}^{n}(x)=0$ for all $x \in X_{n}$.

\footnotetext{
${ }^{3}$ Clearly, if $\mathcal{U} \in \Omega(X)$ and $X$ satisfies $\mathrm{S}_{1}(\Omega, \Gamma)$, then $\mathcal{U}$ contains a subcover $\mathcal{V}$ such that $\mathcal{V} \in \Gamma(X)[13$.

${ }^{4}$ For each $x \in X, x \in X_{n}$ for all large enough $n$. Thus, for all large enough $n, r_{n}(x)=$ $\sup \left\{\left|f_{m}^{n}(x)\right|: m \in I_{n}\right\}$ is defined, and therefore the question as to whether $\lim _{n} r_{n}(x)$ converges to 0 or not makes sense.
} 
Step 1: As $C\left(X_{1}\right)$ is $\alpha_{2}$, there are infinite $J_{1}, J_{2}, \ldots \subseteq \mathbb{N}$ such that any enumeration of the countable set $\bigcup_{n}\left\{f_{m}^{n}(x): m \in J_{n}\right\}$ converges to 0 , for each $x \in X_{1}$. In particular, $\lim _{n} \sup \left\{\left|f_{m}^{n}(x)\right|: m \in J_{n}\right\}=0$ for all $x \in X_{1}$. Let $I_{1}=J_{1}$.

Step $k$ : For each $n \geq k, \lim _{m \in J_{n}} f_{m}^{n}(x)=0$ for all $x \in X_{k}$. As $C\left(X_{k}\right)$ is $\alpha_{2}$, the index sets $J_{k}, J_{k+1}, \ldots$ may be thinned out so that they remain infinite, and $\lim _{n} \sup \left\{\left|f_{m}^{n}(x)\right|: m \in J_{n}\right\}=0$ for all $x \in X_{k}$. Let $I_{k}=J_{k}$.

The index sets $I_{1}, I_{2}, \ldots$ are as required.

Corollary 2.12. Assume that for each subset $Y$ of $X, C(Y)$ is $\alpha_{2}$. For all $X_{1} \subseteq$ $X_{2} \subseteq \ldots \subseteq X, C\left(\bigcup_{n} X_{n}\right)$ is $\left\{X_{n}\right\}_{n \in \mathbb{N}}-\alpha_{2}$.

A topological space is perfectly normal if for each pair of disjoint closed sets $C_{0}, C_{1} \subseteq X$, there is $f \in C(X)$ such that $f^{-1}[\{0\}]=C_{0}$ and $f^{-1}[\{1\}]=C_{1}$.

Corollary 2.13. Assume that $X$ is a perfectly normal space and $C(X)$ is $\alpha_{2}$. For all closed sets $X_{1} \subseteq X_{2} \subseteq \ldots \subseteq X, C\left(\bigcup_{n} X_{n}\right)$ is $\left\{X_{n}\right\}_{n \in \mathbb{N}}-\alpha_{2}$.

Proof. If $C(X)$ is $\alpha_{2}$ and $Y$ is a closed subset of $X$, then $C(Y)$ is $\alpha_{2} \cdot 5$ Apply Theorem 2.11.

In addition to these applications, Jordan's Lemma 2.9 is an ingredient in a solution of a thus far open problem, to which we now turn.

\section{3. $\gamma$-SETS OF REALS FROM A WEAK HYPOTHESIS}

In this section, we construct sets of reals satisfying $\mathrm{S}_{1}(\Omega, \Gamma)$. Traditionally, general topological spaces satisfying $\mathrm{S}_{1}(\Omega, \Gamma)$ are called $\gamma$-spaces, and if they happen to be (homeomorphic to) sets of real numbers, they are called $\gamma$-sets.

The problem settled by our construction has some history, which we now survey briefly. This involves combinatorial cardinal characteristics of the continuum 4 . We give the necessary definitions as we proceed. Readers who are new to this field may skip this section in their first reading.

$\gamma$-spaces were introduced by Gerlits and Nagy in [13, their most influential paper, as the third property in a list numbered $\alpha$ through $\epsilon$. This turned out to be the most important property in the list and obtained its alphabetic number as its name. One of the main results in [13] is that for Tychonoff spaces $X, C(X)$ with the topology of pointwise convergence is Fréchet-Urysohn if, and only if, $X$ is a $\gamma$-space.

While uncountable $\gamma$-spaces exist in ZFC6 [30, Borel's Conjecture (which is consistent with, but not provable within, ZFC) implies that all metrizable $\gamma$-spaces are countable.

Since we are dealing with constructions rather than general results, we restrict our attention in this section to subsets of $\mathbb{R}$ (or, since the property is preserved by continuous images, subsets of any topological space which can be embedded in $\mathbb{R}$ ).

\footnotetext{
${ }^{5}$ The argument is as in Theorem 4.1 of [7]: As $X$ is perfectly normal, the open set $X \backslash Y$ is a countable increasing union of closed sets, $X \backslash Y=\bigcup_{n} C_{n}$. For each $n$, extend each $f_{m}^{n}$ to an element of $C(X)$ which is constantly 0 on $C_{n}$. Applying $\alpha_{2}$ on the new sequences, we obtain $m_{n}$ such that $\lim _{n} f_{m_{n}}^{n}(x)=0$ for all $x \in X$, and thus this is also the case for the original functions, for all $x \in Y$.

${ }^{6}$ The axioms of Zermelo and Fraenkel, together with the axiom of choice, the ordinary axioms of mathematics.
} 
Thus, we may restrict our attention to countable open covers. As mentioned above, by a $\gamma$-set we mean a $\gamma$-space which is (homeomorphic to) a set of real numbers.

Gerlits and Nagy proved in [13] that Martin's Axiom implies that all sets of reals of cardinality less than $\mathfrak{c}$ are $\gamma$-sets. There is a simple reason for this: The critical cardinality of a property $P$, denoted non $(P)$, is the minimal cardinality of a set not satisfying $P$. Let $\left(\begin{array}{l}\Omega \\ \Gamma\end{array}\right)$ be the property: Each $\mathcal{U} \in \Omega(X)$ contains a set $\mathcal{V} \in \Gamma(X)$. Gerlits and Nagy proved that $\mathrm{S}_{1}(\Omega, \Gamma)=\left(\begin{array}{c}\Omega \\ \Gamma\end{array}\right)\left[13\right.$. Let $A \subseteq \subseteq^{*} B$ mean that $A \backslash B$ is finite. $A$ is a pseudointersection of $\mathcal{F}$ if $A \subseteq^{*} B$ for all $B \in \mathcal{F}$. Let $\mathfrak{p}$ be the minimal cardinality of a family $\mathcal{F}$ of infinite subsets of $\mathbb{N}$ which is closed under finite intersections and has no pseudointersection. Then non $\left(\begin{array}{l}\Omega \\ \Gamma\end{array}\right)=\mathfrak{p}[12$, and Martin's Axiom implies $\mathfrak{p}=\mathfrak{c}[12$.

By definition, for each property $P$ of sets of reals, every set of reals whose cardinality is smaller than $\operatorname{non}(P)$ satisfies $P$. Thus, the real question is whether there is a set of reals $X$ of cardinality at least non $(P)$ which satisfies $P$. Galvin and Miller [12] proved a result of this type: $\mathfrak{p}=\mathfrak{c}$ implies that there is a $\gamma$-set of cardinality $\mathfrak{p}$. Just, Miller, Scheepers and Szeptycki [17] have improved the construction of [12]. We introduce their construction in a slightly more general form that will be useful later.

Cantor's space $\{0,1\}^{\mathbb{N}}$ is equipped with the Tychonoff product topology, and $P(\mathbb{N})$ is identified with $\{0,1\}^{\mathbb{N}}$ using characteristic functions. This defines the topology of $P(\mathbb{N})$. The partition $P(\mathbb{N})=[\mathbb{N}]^{\infty} \cup[\mathbb{N}]^{<\infty}$, into the infinite and the finite sets, respectively, is useful here.

For $f, g \in \mathbb{N}^{\mathbb{N}}$, let $f \leq^{*} g$ if $f(n) \leq g(n)$ for all but finitely many $n$. $\mathfrak{b}$ is the minimal cardinality of a $\leq^{*}$-unbounded subset of $\mathbb{N}^{\mathbb{N}}$. A set $B \subseteq[\mathbb{N}]^{\infty}$ is unbounded if the set of all increasing enumerations of elements of $B$ is unbounded in $\mathbb{N}^{\mathbb{N}}$, with respect to $\leq^{*}$. It follows that $|B| \geq \mathfrak{b}$. For $m, n \in \mathbb{N}$, let $(m, n)=\{k: m<k<n\}$.

Lemma 3.1 (Folklore). If $B \subseteq[\mathbb{N}]^{\infty}$ is unbounded, then for each increasing $f \in$ $\mathbb{N}^{\mathbb{N}}$, there is $x \in B$ such that $x \cap(f(n), f(n+1))=\emptyset$ for infinitely many $n$.

Proof. Assume that $f$ is a counterexample. Let $g$ dominate all functions $f_{m}(n)=$ $f(n+m), m \in \mathbb{N}$. Then for each $x \in B, x \leq^{*} g$. Indeed, let $m$ be such that for all $n \geq m, x \cap(f(n), f(n+1)) \neq \emptyset$. Then for each $n$, the $n$-th element of $x$ is smaller than $f_{m+1}(n)$.

Definition 3.2. Atower of cardinality $\kappa$ is a set $T \subseteq[\mathbb{N}]^{\infty}$ which can be enumerated bijectively as $\left\{x_{\alpha}: \alpha<\kappa\right\}$, such that for all $\alpha<\beta<\kappa, x_{\beta} \subseteq \subseteq^{*} x_{\alpha}$.

An unbounded tower of cardinality $\kappa$ is an unbounded set $T \subseteq[\mathbb{N}]^{\infty}$ which is a tower of cardinality $\kappa$. (Necessarily, $\kappa \geq \mathfrak{b}$.)

Let $\mathfrak{t}$ be the minimal cardinality of a tower which has no pseudointersection. Rothberger proved that $\mathfrak{t} \leq \mathfrak{b}[4$.

Lemma 3.3 (Folklore). $\mathfrak{t}=\mathfrak{b}$ if, and only if, there is an unbounded tower of cardinality $\mathfrak{t}$.

Proof. $(\Rightarrow)$ Construct $x_{\alpha}$ by induction on $\alpha$. Let $\left\{b_{\alpha}: \alpha<\mathfrak{b}\right\} \subseteq \mathbb{N}^{\mathbb{N}}$ be unbounded. At step $\alpha$, let $a$ be a pseudointersection of $\left\{x_{\beta}: \beta<\alpha\right\}$, and take $x_{\alpha} \subseteq a$ such that the increasing enumeration of $x_{\alpha}$ dominates $b_{\alpha}$.

$(\Leftarrow) \mathfrak{t} \leq \mathfrak{b} \leq|T|=\mathfrak{t}$.

Just, Miller, Scheepers and Szeptycki [17] proved that if $T$ is an unbounded tower of cardinality $\aleph_{1}$, then $T \cup[\mathbb{N}]^{<\infty}$ satisfies $\mathrm{S}_{1}(\Omega, \Omega)$, as well as a property, which 
was later proved by Scheepers [26] to be equivalent to $S_{1}(\Gamma, \Gamma)$. In Problem 7 of [17, we are asked the following.

Problem 3.4 (Just-Miller-Scheepers-Szeptycki [17). Assume that $T \subseteq[\mathbb{N}]^{\infty}$ is an unbounded tower of cardinality $\aleph_{1}$ (so that $\aleph_{1}=\mathfrak{b}$ ). Is $T \cup[\mathbb{N}]<\infty$ a $\gamma$-set, i.e., does it satisfy $\mathrm{S}_{1}(\Omega, \Gamma)$ ?

Scheepers proves in 25] that for each unbounded tower $T$ of cardinality $\mathfrak{t}=\mathfrak{b}$, $T \subseteq[\mathbb{N}]^{\infty}$ satisfies $\mathrm{S}_{1}(\Gamma, \Gamma)$.

Miller 21 proves that in the Hechler model there are no uncountable $\gamma$-sets. In this model, $\aleph_{1}=\mathfrak{p}=\mathfrak{t}<\mathfrak{b}$, and thus $\aleph_{1}=\mathfrak{t}$ does not suffice to have an uncountable $\gamma$-set. At the end of 21] and in its appendix, Miller proves that $\diamond(\mathfrak{b})$, a property strictly stronger than $\aleph_{1}=\mathfrak{b}$, implies that there is an uncountable $\gamma$ set 7 He concludes that it is still an open question as to whether $\mathfrak{b}=\aleph_{1}$ is enough to construct an uncountable $\gamma$-set.

We show that the answer is positive, and indeed also answer a question of Gruenhage and Szeptycki [14: A classical problem of Malykhin asks whether there is a countable Fréchet-Urysohn topological group which is not metrizable. Gruenhage and Szeptycki prove that $F \subseteq \mathbb{N}^{\mathbb{N}}$ is a $\gamma$-set if, and only if, a certain construction associated to $F$ provides a positive answer to Malykhin's Problem [14. They define a generalization of the $\gamma$-set, called a weak $\gamma$-set, and combine their results with results of Nyikos to prove the following.

Theorem 3.5 (Gruenhage-Szeptycki [14]). If $\mathfrak{p}=\mathfrak{b}$, then there is a weak $\gamma$-set in $\mathbb{N}^{\mathbb{N}}$.

They write: "The relationship between $\gamma$-sets and weak $\gamma$-sets is not known. Perhaps $\mathfrak{b}=\mathfrak{p}$ implies the existence of a $\gamma$-set" [14. Our solution confirms their conjecture.

$\mathfrak{p} \leq \mathfrak{t} \leq \mathfrak{b}$, and in all known models of set theory, $\mathfrak{p}=\mathfrak{t}$. When $\mathfrak{p}=\mathfrak{c}$, our theorem shows that in Galvin and Miller's construction from [12, even if the possible open covers are not considered at all, the resulting set is still a $\gamma$-set.

Theorem 3.6. For each unbounded tower $T$ of cardinality $\mathfrak{p}$ in $[\mathbb{N}]^{\infty}, T \cup[\mathbb{N}]^{<\infty}$ satisfies $\mathrm{S}_{1}(\Omega, \Gamma)$.

Proof. By Lemma 3.3, there is an unbounded tower $T$ of cardinality $\mathfrak{p}$ if, and only if, $\mathfrak{p}=\mathfrak{b}$. Let $T=\left\{x_{\alpha}: \alpha<\mathfrak{b}\right\}$ be an unbounded tower of cardinality $\mathfrak{b}$. For each $\alpha$, let $X_{\alpha}=\left\{x_{\beta}: \beta<\alpha\right\} \cup[\mathbb{N}]^{<\infty}$. We will show that $T \cup[\mathbb{N}]^{<\infty}$ satisfies $\left(\begin{array}{c}\Omega \\ \Gamma\end{array}\right)$. Let $\mathcal{U} \in \Omega\left(T \cup[\mathbb{N}]^{<\infty}\right)$. We use the following modification of Lemma 1.2 of [12].

Lemma 3.7. Assume that $[\mathbb{N}]^{<\infty} \subseteq X \subseteq P(\mathbb{N})$ and $X$ satisfies $\left(\begin{array}{c}\Omega \\ \Gamma\end{array}\right)$. For each $\mathcal{U} \in \Omega(X)$, there are $m_{1}<m_{2}<\ldots$ and distinct $U_{1}, U_{2}, \ldots \in \mathcal{U}$ such that $\left\{U_{n}\right.$ : $n \in \mathbb{N}\} \in \Gamma(X)$, and for each $x \subseteq \mathbb{N}, x \in U_{n}$ whenever $x \cap\left(m_{n}, m_{n+1}\right)=\emptyset$.

Proof. As $X$ satisfies $\left(\begin{array}{l}\Omega \\ \Gamma\end{array}\right)$, we may thin out $\mathcal{U}$ so that $\mathcal{U} \in \Gamma(X)$.

We proceed as in the proof of Lemma 1.2 of [12]. Let $m_{1}=1$. For each $n \geq 1$ : As $\mathcal{U} \in \Omega(X)$, each finite subset of $X$ is contained in infinitely many elements of $\mathcal{U}$. Take $U_{n} \in \mathcal{U} \backslash\left\{U_{1}, \ldots, U_{n-1}\right\}$, such that $P\left(\left\{1, \ldots, m_{n}\right\}\right) \subseteq U_{n}$. As $U_{n}$ is open, for each $s \subseteq\left\{1, \ldots, m_{n}\right\}$ there is $k_{s}$ such that for each $x \in P(\mathbb{N})$ with $x \cap\left\{1, \ldots, k_{s}-1\right\}=s, x \in U_{n}$. Let $m_{n+1}=\max \left\{k_{s}: s \subseteq\left\{1, \ldots, m_{n}\right\}\right\}$.

\footnotetext{
${ }^{7} \diamond(\mathfrak{b})$ is defined in Dzamonja-Hrusak-Moore [10].
} 
As $\left\{U_{n}: n \in \mathbb{N}\right\}$ is an infinite subset of $\mathcal{U} \in \Gamma(X),\left\{U_{n}: n \in \mathbb{N}\right\} \in \Gamma(X)$, too.

We may assume that $\mathcal{U}$ is countable. As $\mathfrak{b}$ is regular, there is $\alpha_{1}<\mathfrak{b}$ such that $X_{\alpha_{1}}$ is not contained in any member of $\mathcal{U}$. This guarantees that $\mathcal{U} \in \Omega\left(X_{\alpha}\right)$ for all $\alpha \geq \alpha_{1}$.

As $\left|X_{\alpha_{1}}\right|<\mathfrak{p}, X_{\alpha_{1}}$ satisfies $\left(\begin{array}{c}\Omega \\ \Gamma\end{array}\right)$. As $\mathcal{U} \in \Omega\left(X_{\alpha_{1}}\right)$, by Lemma 3.7 there are $m_{1}^{1}<m_{2}^{1}<\ldots$ and distinct $U_{1}^{1}, U_{2}^{1}, \ldots \in \mathcal{U}$ such that $\left\{U_{n}^{1}: n \in \mathbb{N}\right\} \in \Gamma\left(X_{\alpha_{1}}\right)$, and for each $x \in P(\mathbb{N}), x \in U_{n}^{1}$ whenever $x \cap\left(m_{n}^{1}, m_{n+1}^{1}\right)=\emptyset$. Let $D_{1}=\mathbb{N}$.

As $\alpha_{1}<\mathfrak{b},\left\{x_{\alpha}: \alpha_{1}<\alpha<\mathfrak{b}\right\}$ is unbounded. By Lemma 3.1, there is $\alpha_{2}>\alpha_{1}$ such that $D_{2}=\left\{n: x_{\alpha_{2}} \cap\left(m_{n}^{1}, m_{n+1}^{1}\right)=\emptyset\right\}$ is infinite. As $\left|X_{\alpha_{2}}\right|<\mathfrak{p}, X_{\alpha_{2}}$ satisfies $\left(\begin{array}{c}\Omega \\ \Gamma\end{array}\right)$. As $\mathcal{U} \in \Omega\left(X_{\alpha_{2}}\right)$, by Lemma 3.7 there are $m_{1}^{2}<m_{2}^{2}<\ldots$ and distinct $U_{1}^{2}, U_{2}^{2}, \ldots \in \mathcal{U}$ such that $\left\{U_{n}^{2}: n \in \mathbb{N}\right\} \in \Gamma\left(X_{\alpha_{2}}\right)$, and for each $x \in P(\mathbb{N}), x \in U_{n}^{2}$ whenever $x \cap\left(m_{n}^{2}, m_{n+1}^{2}\right)=\emptyset$. As $D_{2}$ is infinite, $\left\{U_{n}^{2}: n \in D_{2}\right\} \in \Gamma\left(X_{\alpha_{2}}\right)$.

Continue in the same manner to define for each $k>1$ elements with the following properties:

(1) $\alpha_{k}>\alpha_{k-1}$;

(2) $D_{k}=\left\{n: x_{\alpha_{k}} \cap\left(m_{n}^{k-1}, m_{n+1}^{k-1}\right)=\emptyset\right\}$ is infinite;

(3) $m_{1}^{k}<m_{2}^{k}<\ldots$;

(4) $U_{1}^{k}, U_{2}^{k}, \ldots \in \mathcal{U}$ are distinct;

(5) $\left\{U_{n}^{k}: n \in D_{k}\right\} \in \Gamma\left(X_{\alpha_{k}}\right)$; and

(6) for each $x \in P(\mathbb{N}), x \in U_{n}^{k}$ whenever $x \cap\left(m_{n}^{k}, m_{n+1}^{k}\right)=\emptyset$.

Let $\alpha=\sup _{k} \alpha_{k}$. As $\mathfrak{b}$ is regular, $\alpha<\mathfrak{b} . X_{\alpha}=\bigcup_{k} X_{\alpha_{k}}$ is a countable increasing union. For each $k,\left|X_{\alpha_{k}}\right|<\mathfrak{b}$, and thus $X_{\alpha_{k}}$ satisfies $\mathrm{S}_{1}(\Gamma, \Gamma)$. By Lemma 2.9, there are infinite $I_{1} \subseteq D_{1}, I_{2} \subseteq D_{2}, \ldots$ such that each $x \in X_{\alpha}$ belongs to $\bigcap_{n \in I_{k}} U_{n}^{k}$ for all but finitely many $k \in \mathbb{N}$.

Take $n_{1} \in I_{2}$. For $k>1$, take $n_{k} \in I_{k+1}$ such that $m_{n_{k}}^{k}>m_{n_{k-1}+1}^{k-1}, x_{\alpha} \cap$ $\left(m_{n_{k}}^{k}, m_{n_{k}+1}^{k}\right) \subseteq x_{\alpha_{k+1}} \cap\left(m_{n_{k}}^{k}, m_{n_{k}+1}^{k}\right)$, and $U_{n_{k}}^{k} \notin\left\{U_{n_{1}}^{1}, \ldots, U_{n_{k-1}}^{k-1}\right\}$. We claim that $\left\{U_{n_{k}}^{k}: k \in \mathbb{N}\right\} \in \Gamma\left(T \cup[\mathbb{N}]^{<\infty}\right)$. As $\left\{U_{n_{k}}^{k}: k \in \mathbb{N}\right\} \in \Gamma\left(X_{\alpha}\right)$, it remains to show that for each $x \subseteq^{*} x_{\alpha}, x \in U_{n_{k}}^{k}$ for all but finitely many $k$. For each large enough $k, m_{n_{k}}^{k}$ is large enough, so that

$$
x \cap\left(m_{n_{k}}^{k}, m_{n_{k}+1}^{k}\right) \subseteq x_{\alpha} \cap\left(m_{n_{k}}^{k}, m_{n_{k}+1}^{k}\right) \subseteq x_{\alpha_{k+1}} \cap\left(m_{n_{k}}^{k}, m_{n_{k}+1}^{k}\right)=\emptyset,
$$

since $n_{k} \in D_{k+1}$. Thus, $x \in U_{n_{k}}^{k}$.

Remark 3.8. Zdomskyy points out that our proof actually shows that a wider family of sets are $\gamma$-sets. For example, if we start with $T$ an unbounded tower of cardinality $\mathfrak{p}$, and thin out its elements arbitrarily, $T \cup[\mathbb{N}]<\infty$ remains a $\gamma$-set. This may be useful for constructions of examples with additional properties, since this way each element of $T$ may be chosen arbitrarily from a certain perfect set.

In particular, we have that in each model of ZFC where $\mathfrak{p}=\mathfrak{b}$, there are $\gamma$-sets of cardinality $\mathfrak{p}$. In the following corollary, by an " $X$ model" we mean the model obtained by generically extending a model of the Continuum Hypothesis by adding $\aleph_{2}$ (or more, in the case $X \in\{$ Cohen, Random $\}$ ) $X$ reals in the standard way; see [4] for details.

Corollary 3.9. In each of the Cohen, Random, Sacks, and Miller models of ZFC, there are $\gamma$-sets of reals with cardinality $\mathfrak{p}$. 
Earlier, Corollary 3.9 was shown for the Sacks model by Ciesielski, Millán, and Pawlikowski in [9], and for the Cohen and Miller models by Miller [21, using specialized arguments. It seems that the result, that there are uncountable $\gamma$-sets in the random reals model (constructed by extending a model of the Continuum Hypothesis), is new. We point out that the Random poset alone is not the reason for having uncountable $\gamma$-sets in the generic extension: Judah, Shelah, and Woodin prove in [16] that there are no uncountable strong measure zero sets (and in particular, no uncountable $\gamma$-sets) in an extension of Laver's model by random reals.

As discussed above, there are no uncountable $\gamma$-sets in the Hechler model 21 . Since the Laver and Mathias models satisfy Borel's Conjecture, there are no uncountable $\gamma$-sets in these models either.

\section{HEREDITY}

A topological space $X$ satisfies a property $P$ hereditarily if each subspace of $X$ satisfies $P$. In our context, heredity was observed to be tightly connected to the following property. $X$ is a $\sigma$-space if each Borel subset of $X$ is an $F_{\sigma}$ subset of $X$.

A combination of results of Fremlin-Miller [11, Bukovský-Recław-Repický [8, and Bukovský-Haleš [6] implies that a Tychonoff space $X$ satisfies $\mathrm{U}_{\text {fin }}(\mathrm{O}, \Gamma)$ hereditarily if, and only if, $X$ satisfies $\mathrm{U}_{\text {fin }}(\mathrm{O}, \Gamma)$ and is a $\sigma$-space (see also [37) 8 A similar result was proved for $S_{1}(\Gamma, \Gamma)$ in [6]. Problem 7.9 in 8 ] asks whether every (nice enough) $\sigma$-space $X$ satisfying $\mathrm{S}_{1}(\Omega, \Gamma)$ satisfies $\mathrm{S}_{1}(\Omega, \Gamma)$ hereditarily. A negative answer was given by Miller [20.

We have, in light of the previous section, a simple reason for the difference between $S_{1}(\Omega, \Gamma)$ on one hand, and $S_{1}(\Gamma, \Gamma)$ and $U_{f i n}(O, \Gamma)$ on the other hand.

Definition 4.1. Let $\mathscr{A} \in\{\Gamma, \Omega, \mathrm{O}, \ldots\} . \mathscr{A}$ is Borel superset covering for $X$ if, for each subspace $Y \subseteq X$ and each $\mathcal{U} \in \mathscr{A}(Y)$, there are $\mathcal{V} \subseteq \mathcal{U}$ and a Borel $B \subseteq X$, such that $Y \subseteq B$ and $\mathcal{V} \in \mathscr{A}(B)$.

Many classical types of covers are Borel superset covering. In particular, we have the following.

Lemma 4.2. $\Gamma$ and $\mathrm{O}$ are Borel superset covering (for all $X$ ).

Proof. If $\mathcal{U} \in \Gamma(X)$, take a countable infinite subset $\mathcal{V}=\left\{U_{n}: n \in \mathbb{N}\right\} \subseteq \mathcal{U}$, and $B=\bigcup_{m} \bigcap_{n \geq m} U_{n}$. If $\mathcal{U} \in \mathrm{O}(X)$, take $B=\bigcup \mathcal{U}$.

By Miller's mentioned result and the following theorem, $\Omega$ need not be Borel superset covering.

Theorem 4.3. Let $\mathscr{A}, \mathscr{B} \in\{\Gamma, \Omega, \mathrm{O}\}$ and $\Pi \in\left\{\mathrm{S}_{1}, \mathrm{~S}_{\mathrm{fin}}, \mathrm{U}_{\text {fin }}\right\}$. Assume that $X$ is a $\sigma$-space and satisfies $\Pi(\mathscr{A}, \mathscr{B})$, and $\mathscr{A}$ is Borel superset covering for $X$. Then $X$ satisfies $\Pi(\mathscr{A}, \mathscr{B})$ hereditarily.

Proof. Let $Y \subseteq X$ and assume that $\mathcal{U}_{1}, \mathcal{U}_{2}, \ldots \in \mathscr{A}(Y)$. For each $n$, pick intermediate Borel sets $Y \subseteq B_{n} \subseteq X$ such that $\mathcal{U}_{n} \in \mathscr{A}\left(B_{n}\right)$, and let $B=\bigcap_{n} B_{n}$. Then

\footnotetext{
${ }^{8}$ The assumptions on $X$ in the cited references are stronger, but: A Tychonoff hereditarily$\mathrm{U}_{\mathrm{fin}}(\mathrm{O}, \Gamma)$ space cannot have the unit interval $[0,1]$ as a continuous image, and thus is zerodimensional. As it is hereditarily Lindelöf, each open set is a countable union of clopen sets, and this suffices for the arguments in the cited references.
} 
$\mathcal{U}_{1}, \mathcal{U}_{2}, \ldots \in \mathscr{A}(B)$. As $B$ is Borel and $X$ is a $\sigma$-space, $B$ is $F_{\sigma}$ in $X$. By Corollary 2.4 $B$ satisfies $\Pi(\mathscr{A}, \mathscr{B})$, and we can thus obtain from the covers $\mathcal{U}_{1}, \mathcal{U}_{2}, \ldots$ a cover $\mathcal{V} \in \mathscr{B}(B)$. Then $\mathcal{V} \in \mathscr{B}(Y)$.

As for the other direction, we have, by the discussion at the beginning of this section, the following.

Lemma 4.4. If a property $P$ of Tychonoff spaces implies $\bigcup_{\text {fin }}(\mathrm{O}, \Gamma)$ and $X$ satisfies $P$ hereditarily, then $X$ is a $\sigma$-space.

During his work with the second author on [37, L. Zdomskyy asked whether every subset of $\mathbb{R}$ satisfying $\mathrm{U}_{\text {fin }}(\mathrm{O}, \Omega)$ hereditarily is a $\sigma$-space. We show that the answer is "no", in a very strong sense. We will see that, in the context of Scheepers Diagram, Lemma 4.4 becomes a criterion; that is, satisfying $P$ hereditarily implies being a $\sigma$-space if, and only if, $P$ implies $\mathrm{U}_{\text {fin }}(\mathrm{O}, \Gamma)$.

We use, in our proof, the method of "forcing", following an elegant approach of Brendle [5]. A proof avoiding this method (e.g., using the methods of 32]) would be more lengthy and technically involved.

Theorem 4.5. Assume the Continuum Hypothesis. There is $L \subseteq \mathbb{R}$ such that all finite powers of $L$ satisfy $\mathrm{S}_{1}\left(\mathrm{~B}_{\Omega}, \mathrm{B}_{\Omega}\right)$ hereditarily, but $L$ is not a $\sigma$-space.

Proof. Let $M_{\alpha}, \alpha<\aleph_{1}$, be an increasing sequence of countable submodels of $H(\lambda)$ ( $\lambda$ large enough) such that:

(1) $\mathbb{R} \subseteq \bigcup_{\alpha<\aleph_{1}} M_{\alpha}$;

(2) for each $\alpha<\aleph_{1}, M_{\alpha+1} \models M_{\alpha}$ is countable; and

(3) $\left(M_{\beta}: \beta \leq \alpha\right) \in M_{\alpha+1}$.

For each $\alpha$ choose a real $c_{\alpha}$ Cohen generic over $M_{\alpha}$. (As $M_{\alpha}$ is countable, there is such $c_{\alpha}$.) Let $L=\left\{c_{\alpha}: \alpha<\aleph_{1}\right\}$. It is well known that a set $L$ constructed this way is a Luzin set, i.e., $L$ is uncountable, but for each meager $M \subseteq \mathbb{R}, L \cap M$ is countable 9

Lemma 4.6 (Folklore). No Luzin set $L$ is a $\sigma$-space.

Proof. Assume otherwise, and take a countable dense $D \subseteq L$. As $L \backslash D$ is Borel, it is $F_{\sigma}$. Then $D=\bigcap_{n} U_{n}$, an intersection of open sets. Then for each $n, L \backslash U_{n}$ is meager, and thus countable. It follows that $L \backslash D$ is countable, a contradiction.

Recall that according to Definition 1.2, $X$ satisfies $P^{\uparrow}$ if all finite powers of $X$ satisfy $P$. By [3], or alternatively Lemma 4.2] and Remark 4.9, $\mathrm{S}_{1}(\mathrm{~B}, \mathrm{~B})$ is hereditary. By [28], $\mathrm{S}_{1}\left(\mathrm{~B}_{\Omega}, \mathrm{B}_{\Omega}\right)^{\uparrow}=\mathrm{S}_{1}(\mathrm{~B}, \mathrm{~B})^{\uparrow}$.

Lemma 4.7. If $P$ is hereditary, then so is $P^{\uparrow}$.

Proof. If $Y \subseteq X$ and $X$ satisfies $P^{\uparrow}$, then for each $k, Y^{k} \subseteq X^{k}$ and $X^{k}$ satisfies $P$. Thus, $Y^{k}$ satisfies $P$ for all $k$.

Thus, it suffices to prove that all finite powers of $L$ satisfy $\mathrm{S}_{1}(\mathrm{~B}, \mathrm{~B})$. Consider for example $L^{2}$. We have to show (essentially, 28]) that for each Borel $\Psi: \mathbb{R}^{2} \rightarrow \mathbb{N}^{\mathbb{N}}$, there is $g \in \mathbb{N}^{\mathbb{N}}$ such that for each $(x, y) \in L^{2}, \Psi(x, y)(n)=g(n)$ for some $n$.

Let $\Psi: \mathbb{R}^{2} \rightarrow \mathbb{N}^{\mathbb{N}}$ be Borel. $\Psi$ is coded by a real. Let $\alpha$ be such that this code belongs to $M_{\alpha} . \mathbb{N}^{\mathbb{N}} \cap M_{\alpha}$ is countable. Take a canonical partition $\mathbb{N}=\bigcup_{n} I_{n}$ with

\footnotetext{
${ }^{9}$ Indeed, each meager set is contained in a meager $F_{\sigma}$ set $M$, which in turn is coded by a single real $r$. Let $\alpha$ be such that $r \in M_{\alpha}$. Then for each $\beta>\alpha, c_{\beta} \notin M$.
} 
each $I_{n}$ infinite, so that $\left(I_{n}: n \in \mathbb{N}\right) \in M_{\alpha}$. Enumerate $\mathbb{N}^{\mathbb{N}} \cap M_{\alpha}=\left\{f_{n}: n \in \mathbb{N}\right\}$, and take $g \in \mathbb{N}^{\mathbb{N}}$ such that for each $n,\left.g\right|_{I_{n}}=\left.f_{n}\right|_{I_{n}}$. For $x, y \in L, \Psi(x, y) \in$ $M_{\alpha}[x, y]$, an extension of $M_{\alpha}$ by finitely many Cohen reals, which is in fact either $M_{\alpha}$ or an extension of $M_{\alpha}$ by a single Cohen real. As the Cohen forcing does not add eventually different reals (e.g., [2]), there is $n$ such that $\left.f_{n}\right|_{I_{n}}$ coincides with $\left.\Psi(x, y)\right|_{I_{n}}$ on infinitely many values. But $\left.f_{n}\right|_{I_{n}}=\left.g\right|_{I_{n}}$.

We conclude the section with the following characterization, which may be viewed as a necessary revision of Problem 7.9 of Bukovský-Recław-Repický [8], so that it has a (provably) positive answer 10

Theorem 4.8. A Tychonoff space $X$ satisfies $\mathrm{S}_{1}(\Omega, \Gamma)$ hereditarily if, and only if:

(1) $X$ satisfies $\mathrm{S}_{1}(\Omega, \Gamma)$;

(2) $X$ is a $\sigma$-space; and

(3) $\Omega$ is Borel superset covering for $X$.

Proof. Theorem 4.3 provides the "if" part. For the "only if" part, by Lemma 4.4 it remains to prove (3). Let $Y \subseteq X, \mathcal{U} \in \Omega(Y)$. As $Y$ satisfies $\mathrm{S}_{1}(\Omega, \Gamma)$, there are $U_{n} \in \mathcal{U}$ such that $\left\{U_{n}: n \in \mathbb{N}\right\} \in \Gamma(Y)$. Let $B=\bigcup_{m} \bigcap_{n \geq m} U_{n}$. Then $Y \subseteq B$ and $\left\{U_{n}: n \in \mathbb{N}\right\} \in \Omega(B)$.

Remark 4.9 (The case of countable Borel covers). In the Borel case, the properties are hereditary for Borel subsets 28, and thus in Theorem 4.3, there is no need to assume that $X$ is a $\sigma$-space. This explains why some of the Borel properties are hereditary [3] whereas others are not 20, and why none of the open properties is hereditary [3].

Two major open problems concerning $\mathrm{S}_{1}\left(\mathrm{~B}_{\Omega}, \mathrm{B}_{\Omega}\right)$ and $\mathrm{S}_{\mathrm{fin}}\left(\mathrm{B}_{\Omega}, \mathrm{B}_{\Omega}\right)$ are whether these properties are hereditary, and whether they are - like their open variantpreserved by finite powers [3, 35, 20. By Lemma 4.7, the problems are related: If $S_{1}\left(B_{\Omega}, B_{\Omega}\right)$ is preserved by finite powers, then it is equal to $S_{1}(B, B)^{\uparrow}$, which is hereditary. Similarly for $\mathrm{S}_{\text {fin }}\left(\mathrm{B}_{\Omega}, \mathrm{B}_{\Omega}\right)$.

Item (2) may be removed from Theorem 4.8 in the Borel case. On the other hand, $\mathrm{U}_{\text {fin }}\left(\mathrm{B}, \mathrm{B}_{\Gamma}\right)$ is hereditary and (for Lindelöf spaces) implies $\mathrm{U}_{\mathrm{fin}}(\mathrm{O}, \Gamma)$, and thus implies being a $\sigma$-set. Theorem 4.5 completes the picture.

\section{TOPOLOGICAL GROUPS WITH STRONG COMBINATORIAL PROPERTIES}

Problem 10.7 in [35], which is also implicit in Theorem 20 of 32 and in the discussion around it, asks whether the Continuum Hypothesis implies the existence of an uncountable subgroup $G$ of $\mathbb{Z}^{\mathbb{N}}$ satisfying $\mathrm{S}_{1}(\Omega, \Gamma)$. We will show that the answer is "yes", and indeed the weaker hypothesis $\mathfrak{p}=\mathfrak{b}$ suffices to obtain such groups (see the discussion in Section 3). Indeed, Theorem 3.6 gives an uncountable subset of $\mathbb{Z}^{\mathbb{N}}$ satisfying $S_{1}(\Omega, \Gamma)$. We will show that for a wide class of properties $P$, including $\mathrm{S}_{1}(\Omega, \Gamma)$, if $X$ satisfies $P$, then so is the group generated by $X$ in any Tychonoff topological group $G$.

There is some restriction on $P$ : Let $F(X)$ be the free topological group generated by $X$. That is, the group such that each continuous function $f$ from $X$ into a topological group $H$ can be extended uniquely to a continuous homomorphism from $F(X)$ to $H 11$

\footnotetext{
${ }^{10}$ See the discussion at the beginning of the present section.

${ }^{11} \mathrm{~A}$ thorough introduction to free topological groups is available in 29 .
} 
Lemma 5.1. Assume that $P$ is a property of Tychonoff topological spaces, which is hereditary for closed subsets and preserved by homeomorphic images. If for each $X$ satisfying $P, F(X)$ satisfies $P$, then $P=P^{\uparrow}$.

Proof. Assume that $X$ satisfies $P$. Then $F(X)$ satisfies $P$. For each $k, X^{k}$ is homeomorphic to $\left\{x_{1} \cdots x_{k}: x_{1}, \ldots, x_{k} \in X\right\}$, a closed subspace of $F(X)$, and thus satisfies $P$.

Thus, we must consider properties $P$ such that $P=P^{\uparrow}$. For each $Q$ in the Scheepers Diagram, $P=Q^{\uparrow}$ is as required. By the discussion in the introduction [17:

(1) $\mathrm{S}_{1}(\mathrm{O}, \mathrm{O})^{\uparrow}=\mathrm{S}_{1}(\Omega, \Omega)^{\uparrow}=\mathrm{S}_{1}(\Omega, \Omega)$;

(2) $\mathrm{S}_{\text {fin }}(\mathrm{O}, \mathrm{O})^{\uparrow}=\mathrm{U}_{\text {fin }}(\mathrm{O}, \Omega)^{\uparrow}=\mathrm{S}_{\text {fin }}(\Gamma, \Omega)^{\uparrow}=\mathrm{S}_{\text {fin }}(\Omega, \Omega)^{\uparrow}=\mathrm{S}_{\text {fin }}(\Omega, \Omega)$.

Moreover, $\mathrm{S}_{1}(\Omega, \Gamma)^{\uparrow}=\mathrm{S}_{1}(\Omega, \Gamma)$ [12] and $\mathrm{U}_{\text {fin }}(\mathrm{O}, \Gamma)^{\uparrow}$ also has a simple characterization [18]. The properties $\mathrm{S}_{1}(\Gamma, \Gamma)^{\uparrow}, \mathrm{S}_{1}(\Gamma, \Omega)^{\uparrow}$, and $\mathrm{S}_{1}(\Gamma, \mathrm{O})^{\uparrow}$ seem, however, to be unexplored. By the results of [17, each of these properties is strictly stronger than its non- $\uparrow$-ed version.

All $P$ in the Scheepers Diagram have the properties required in the following theorem.

Theorem 5.2. Assume that $P$ is a property of Tychonoff topological spaces, which is hereditary for closed subsets and preserved by continuous images. If $P$ is linearly $\sigma$-additive, then for each $X$ satisfying $P^{\uparrow}$ and each Tychonoff topological group $G$ containing $X$, the group $\langle X\rangle \leq G$ generated by $X$ satisfies $P^{\uparrow}$.

Proof. It is not difficult to prove the following.

Lemma 5.3. Assume that $P$ is a property of topological spaces, which is hereditary for closed subsets and preserved by continuous images, and $P$ is linearly $\sigma$-additive. Then $P^{\uparrow}$ also has these three properties.

Fix distinct $a, b \in X \backslash\{1\}, 1$ being the identity element of $G$. As $\{1, a\},\{a, b\}$ are discrete subspaces of $G, X \times\{1, a\}$ is homeomorphic to $X \times\{a, b\}$. As $X^{2}$ satisfies $P^{\uparrow}$, so does its closed subset $X \times\{a, b\}$, and thus so does $X \times\{1, a\}$. Thus, so does the image $Y$ of $X \times\{1, a\}$ under the continuous map $(x, y) \mapsto x y^{-1} . Y=X \cup X a^{-1}$, and as $a \in X, 1 \in Y$. As $a \in X,\langle X\rangle=\langle Y\rangle$. Thus, we may assume that $1 \in X$.

$(\{1\} \times X) \cup(X \times\{1\})$ is a closed subset of $X^{2}$ which satisfies $P^{\uparrow}$. Thus, $(\{1\} \times$ $X) \cup(X \times\{1\})$ satisfies $P^{\uparrow}$, and therefore so does its image under the same map, $Y=X \cup X^{-1}$. As $\langle Y\rangle=\langle X\rangle$, we may assume that $X=X^{-1}$ and $1 \in X$.

Thus,

$$
\langle X\rangle=\bigcup_{n}\left\{x_{1} \cdots x_{n}: x_{1}, \ldots, x_{n} \in X\right\}
$$

is an increasing union. For each $n,\left\{x_{1} \cdots x_{n}: x_{1}, \ldots, x_{n} \in X\right\}$ is a continuous image of $X^{n}$, which satisfies $P^{\uparrow}$. Thus, $\langle X\rangle$ satisfies $P^{\uparrow}$.

Theorem 5.2 can also be stated in the language of free topological groups. We obtain results analogous (but incomparable) to ones of Banakh, Repovš, and Zdomskyy [1]. 
Theorem 5.4. Let $P$ be as in Theorem 5.2, For each topological space $X$ satisfying $P^{\uparrow}$, the free topological group $F(X)$ satisfies $P^{\uparrow}$.

It follows that for each $P$ in the Scheepers Diagram, and each $X$ satisfying $P^{\uparrow}$, $\langle X\rangle$ satisfies $P^{\uparrow}$. Previous constructions of topological groups satisfying $P^{\uparrow}$ for these properties were much more involved than constructions of topological spaces satisfying $P^{\uparrow}$.

By Theorem 3.6 and Theorem 5.2, we have the following.

Corollary 5.5. Assume that $\mathfrak{p}=\mathfrak{b}$. There is a subgroup of $\mathbb{R}$ of cardinality $\mathfrak{b}$, satisfying $\mathrm{S}_{1}(\Omega, \Gamma)$.

We conclude with an application of the results of this paper. To put it in context, we draw in Figure 2 the Scheepers Diagram, extended to also contain the Borel properties 28. In the Borel case, some additional equivalences hold, and thus there are fewer distinct properties. In particular, $\mathrm{U}_{\text {fin }}\left(\mathrm{B}, \mathrm{B}_{\Gamma}\right)$, the Borel counterpart of $\mathrm{U}_{\text {fin }}(\mathrm{O}, \Gamma)$, is equivalent to $\mathrm{S}_{1}\left(\mathrm{~B}_{\Gamma}, \mathrm{B}_{\Gamma}\right)[28$.

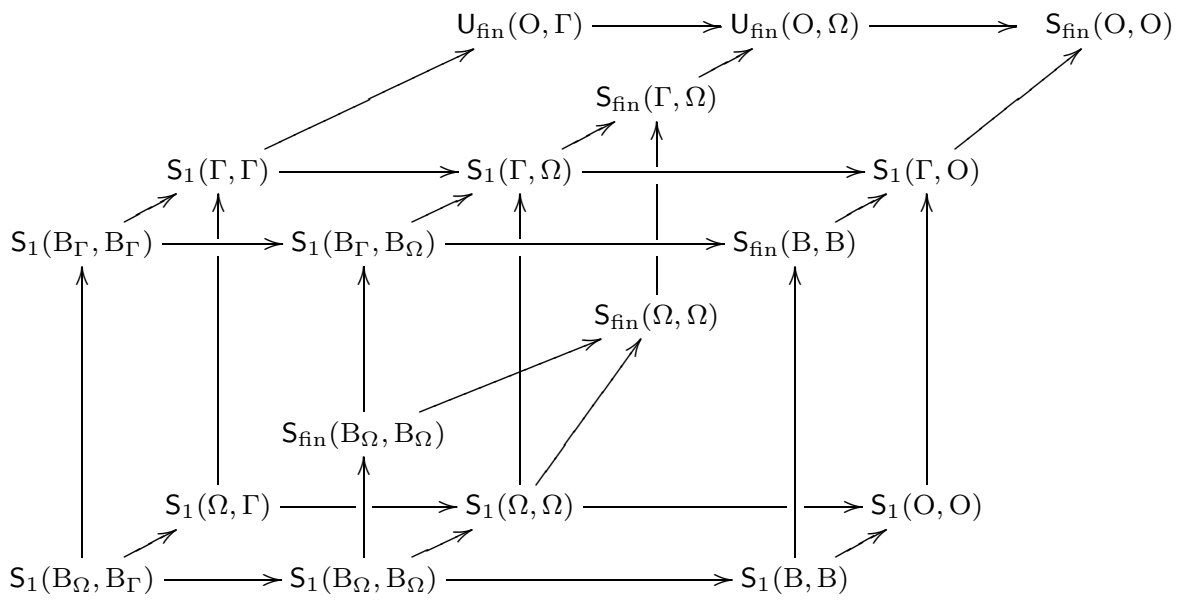

Figure 2. The extended Scheepers Diagram

Theorem 5.6. Assume the Continuum Hypothesis.

(1) There is a subgroup $G$ of $\mathbb{R}$ of cardinality continuum such that each finite power of $G$ satisfies $\mathrm{S}_{1}\left(\mathrm{~B}_{\Omega}, \mathrm{B}_{\Omega}\right)$ hereditarily, but $G$ is not a $\sigma$-space and does not satisfy $\mathrm{U}_{\text {fin }}(\mathrm{O}, \Gamma)$.

(2) There is a subgroup $H$ of $\mathbb{R}$ of cardinality continuum such that all finite powers of $H$ satisfy $\mathrm{S}_{1}\left(\mathrm{~B}_{\Gamma}, \mathrm{B}_{\Gamma}\right)$ and $\mathrm{S}_{\text {fin }}\left(\mathrm{B}_{\Omega}, \mathrm{B}_{\Omega}\right)$ hereditarily, but $H$ does not satisfy $\mathrm{S}_{1}(\mathrm{O}, \mathrm{O})$.

Proof. By [3], or alternatively Lemma 4.2 and Remark 4.9, $\mathrm{S}_{1}(\mathrm{~B}, \mathrm{~B}), \mathrm{S}_{1}\left(\mathrm{~B}_{\Gamma}, \mathrm{B}_{\Gamma}\right)$, and $\mathrm{S}_{\text {fin }}(\mathrm{B}, \mathrm{B})$ are all hereditary. By $28, \mathrm{~S}_{1}\left(\mathrm{~B}_{\Omega}, \mathrm{B}_{\Omega}\right)^{\uparrow}=\mathrm{S}_{1}(\mathrm{~B}, \mathrm{~B})^{\uparrow}$, and similarly for $S_{\text {fin }}$. Thus, by Lemma 4.7 it suffices to prove the statements with the words "hereditarily" removed. 
(1) In the proof of Theorem4.5, we have constructed a Luzin set $L \subseteq \mathbb{R}$ satisfying $\mathrm{S}_{1}\left(\mathrm{~B}_{\Omega}, \mathrm{B}_{\Omega}\right)^{\uparrow} . L$ is not a $\sigma$-space (Lemma 4.6). Let $G=\langle L\rangle$. By Theorem 5.2, $G$ satisfies $\mathrm{S}_{1}\left(\mathrm{~B}_{\Omega}, \mathrm{B}_{\Omega}\right)^{\uparrow}$.

As $L \subseteq G$ and being a $\sigma$-space is hereditary, $G$ is not a $\sigma$-space. As $\mathrm{S}_{1}(\mathrm{~B}, \mathrm{~B})$ is hereditary and is not satisfied by perfect subsets of $\mathbb{R} 12$ does not contain any perfect subset of $\mathbb{R}$. Assume that $G$ satisfies $\mathrm{U}_{\text {fin }}(\mathrm{O}, \Gamma)$. Theorem 5.5 of [17] tells us that sets satisfying $\mathrm{U}_{\text {fin }}(\mathrm{O}, \Gamma)$ and not containing perfect sets are perfectly meager. In particular, $G$ is meager, and thus so is $L$, a contradiction.

(2) By Theorem 23 and Lemma 24 of [32], there is a Sierpiński set $13, S \subseteq \mathbb{R}$ satisfying $\mathrm{S}_{1}\left(\mathrm{~B}_{\Gamma}, \mathrm{B}_{\Gamma}\right)^{\uparrow}$. (This result can alternatively and more easily be proved like Theorem 4.5 by using random reals instead of Cohen reals.) Let $H=\langle S\rangle$. By Theorem [5.2, $H$ satisfies $\mathrm{S}_{1}\left(\mathrm{~B}_{\Gamma}, \mathrm{B}_{\Gamma}\right)^{\uparrow}$. As $\mathrm{S}_{1}\left(\mathrm{~B}_{\Gamma}, \mathrm{B}_{\Gamma}\right)$ implies $\mathrm{S}_{\text {fin }}(\mathrm{B}, \mathrm{B}), \mathrm{U}_{\text {fin }}\left(\mathrm{B}, \mathrm{B}_{\Gamma}\right)^{\uparrow}$ implies $\mathrm{S}_{\text {fin }}(\mathrm{B}, \mathrm{B})^{\uparrow}$, which is $\mathrm{S}_{\text {fin }}\left(\mathrm{B}_{\Omega}, \mathrm{B}_{\Omega}\right)^{\uparrow}$.

It remains to prove that $H$ does not satisfy $\mathrm{S}_{1}(\mathrm{O}, \mathrm{O})$. Assume otherwise. By Remark [4.9, as $H$ satisfies $\mathrm{S}_{1}\left(\mathrm{~B}_{\Gamma}, \mathrm{B}_{\Gamma}\right)$, it is a $\sigma$-space [28]. By Theorem 4.3, its subset $S$ also satisfies $\mathrm{S}_{1}(\mathrm{O}, \mathrm{O})$, and thus has Lebesgue measure zero, a contradiction.

\section{ACKNOWLEDGMENTS}

This work is an extension of a part of the first-named author's M.Sc. thesis at the Weizmann Institute of Science, supervised by Gady Kozma and the second-named author. We thank Gady Kozma for useful discussions and the Weizmann Institute of Science for the stimulating atmosphere. We owe special thanks to Lyubomyr Zdomskyy for reading the paper and making useful comments.

\section{REFERENCES}

[1] T. Banakh, D. Repovš, and L. Zdomskyy, o-Boundedness of free topological groups, Topology Appl. 157 (2010), 466-481. MR2563296 (2010j:54058)

[2] T. Bartoszyński and H. Judah, Set Theory: On the structure of the real line, A. K. Peters, Massachusetts: 1995. MR1350295 (96k:03002)

[3] T. Bartoszyński and B. Tsaban, Hereditary topological diagonalizations and the MengerHurewicz Conjectures, Proceedings of the American Mathematical Society 134 (2006), 605615. MR2176030 (2006f:54038)

[4] A. Blass, Combinatorial cardinal characteristics of the continuum, in: Handbook of Set Theory (M. Foreman, A. Kanamori, and M. Magidor, eds.), Kluwer Academic Publishers, Dordrecht, to appear. http://www.math.lsa.umich.edu/ ablass/hbk.pdf

[5] J. Brendle, Generic constructions of small sets of reals, Topology and its Applications 71 (1996), 125-147. MR1399552 (97i:03030)

[6] L. Bukovský and J. Haleš, On Hurewicz properties, Topology and its Applications 132 (2003), 71-79. MR.1990080 (2004f:03085)

[7] L. Bukovský, I. Recław, and M. Repický, Spaces not distinguishing pointwise and quasinormal convergence of real functions, Topology and its Applications 41 (1991), 25-41. MR.1129696 (93b:54037)

[8] L. Bukovský, I. Recław, and M. Repický, Spaces not distinguishing convergences of real-valued functions, Topology and its Applications 112 (2001), 13-40. MR 1815270 (2002e:54010)

[9] K. Ciesielski, A. Millán, and J. Pawlikowski, Uncountable $\gamma$-sets under axiom CPA $^{\text {game }}{ }^{\text {cube, }}$ Fundamenta Mathematicae 176 (2003), 143-155. MR.1971305 (2004b:03077)

\footnotetext{
${ }^{12}$ Assume that a perfect set satisfies $S_{1}(B, B)$. Then so does the unit interval $[0,1]$, its continuous image. But even $\mathrm{S}_{1}(\mathrm{O}, \mathrm{O})$ implies Lebesgue measure zero.

${ }^{13} S \subseteq \mathbb{R}$ is a Sierpinski set if it is uncountable, but has countable intersection with each Lebesgue measure zero set.
} 
[10] M. Džamonja, M. Hruśak, and J. Moore, Parametrized $\diamond$ principles, Transactions of the American Mathematical Society 356 (2004), 2281-2306. MR2048518 (2005b:03114)

[11] D. Fremlin and A. Miller, On some properties of Hurewicz, Menger and Rothberger, Fundamenta Mathematica 129 (1988), 17-33. MR954892 (89g:54061)

[12] F. Galvin and A. Miller, $\gamma$-sets and other singular sets of real numbers, Topology and its Applications 17 (1984), 145-155. MR738943 (85f:54011)

[13] J. Gerlits and Zs. Nagy, Some properties of $C(X)$. I, Topology and its Applications 14 (1982), 151-161. MR667661 (84f:54021)

[14] Gary Gruenhage and Paul Szeptycki, Fréchet Urysohn for finite sets, Topology and its Applications 151 (2005) 238-259. MR2139755 (2006i:54004)

[15] F. Jordan, There are no hereditary productive $\gamma$-spaces, Topology and its Applications 155 (2008), 1786-1791. MR2445301 (2009f:54029)

[16] H. Judah, S. Shelah, and H. Woodin, The Borel conjecture, Annals of Pure and Applied Logic 50 (1990), 255-269. MR 1086456 (91m:03052)

[17] W. Just, A. Miller, M. Scheepers, and P. Szeptycki, The combinatorics of open covers. II, Topology and its Applications 73 (1996), 241-266. MR.1419798 (98g:03115a)

[18] Lj. Kočinac and M. Scheepers, Combinatorics of open covers. VII. Groupability, Fundamenta Mathematicae 179 (2003), 131-155. MR2029229 (2005c:54020)

[19] Lj. Kočinac, Selected results on selection principles, in: Proceedings of the 3rd Seminar on Geometry and Topology (Sh. Rezapour, ed.), July 15-17, Tabriz, Iran, 2004, 71-104. MR2090207 (2005g:54038)

[20] A. Miller, A nonhereditary Borel-cover $\gamma$-set, Real Analysis Exchange 29 (2003/4), 601-606. MR2083799 (2005g:03079)

[21] A. Miller, The $\gamma$-Borel conjecture, Archive for Mathematical Logic 44 (2005), 425-434. Appendix in http://arxiv.org/math/math.LO/0312308. MR2139770(2005m:03096)

[22] A. Miller, Cardinal characteristic for relative $\gamma$-sets, Topology and its Applications 156 (2009), 872-878. MR2498919

[23] M. Sakai, Property $C^{\prime \prime}$ and function spaces, Proceedings of the American Mathematical Society 104 (1988), 917-919. MR964873 (89m:54004)

[24] M. Scheepers, Combinatorics of open covers I: Ramsey theory, Topology and its Applications 69 (1996), 31-62. MR.1378387 (97h:90123)

[25] M. Scheepers, $C_{p}(X)$ and Arhangel'skiǔ's $\alpha_{i}$ spaces, Topology and its Applications 89 (1998), 265-275. MR1645184 (99g:54018)

[26] M. Scheepers, Sequential convergence in $\mathrm{C}_{p}(X)$ and a covering property, East-West Journal of Mathematics 1 (1999), 207-214. MR.1727383 (2000i:54014)

[27] M. Scheepers, Selection principles and covering properties in topology, Note di Matematica 22 (2003), 3-41. MR 2112729 (2005h:54024)

[28] M. Scheepers and B. Tsaban, The combinatorics of Borel covers, Topology and its Applications 121 (2002), 357-382. MR.1908999 (2003e:03091)

[29] M. Tkachenko, Topological groups for topologists. II, Boletín de la Sociedad Matemática Mexicana. Tercera Serie 6 (2000), 1-41. MR.1768506 (2001g:22004)

[30] S. Todorčević, Aronszajn orderings, Duro Kurepa memorial volume, Publications de l'Institut Mathématique (new series) 57 (1995), 29-46. MR1387352 (98g:03116)

[31] B. Tsaban, Some new directions in infinite-combinatorial topology, Set Theory, J. Bagaria and S. Todorčevic, eds., Trends in Mathematics, Birkhäuser (2006), 225-255. MR2267150 (2007f:03064)

[32] B. Tsaban, o-bounded groups and other topological groups with strong combinatorial properties, Proceedings of the American Mathematical Society 134 (2006), 881-891. MR2180906 (2006h:54034)

[33] B. Tsaban, Additivity numbers of covering properties, Selection Principles and Covering Properties in Topology (L. Kočinac, ed.), Quaderni di Matematica 18, Seconda Universita di Napoli, Caserta (2006), 245-282. MR2395757 (2009e:03088)

[34] B. Tsaban, A new selection principle, Topology Proceedings 31 (2007), 319-329. MR2363173 (2008j:54017)

[35] B. Tsaban, Selection principles and special sets of reals, Open Problems in Topology II, E. Pearl, ed., Elsevier B.V. (2007), 91-108. 
[36] B. Tsaban and L. Zdomskyy, Combinatorial images of sets of reals and semifilter trichotomy, Journal of Symbolic Logic, 73 (2008), 1278-1288. MR2467216 (2009h:03064)

[37] B. Tsaban and L. Zdomskyy, Hereditarily Hurewicz spaces and Arhangel'skiu sheaf amalgamations, Journal of the European Mathematical Society, to appear.

Department of Mathematics, Weizmann Institute of Science, Rehovot 76100, Israel E-mail address: talo@weizmann.ac.il

$U R L$ : http://www.orenshtein.com

Department of Mathematics, Bar-Ilan University, Ramat-Gan 52900, Israel

E-mail address: tsaban@math.biu.ac.il

$U R L$ : http://www.cs.biu.ac.il/ tsaban 